\title{
Anterior Middle Superior Alveolar Nerve Block Efficacy Utilizing different Anesthetic Solutions, Reduced Dosage and Conventional Syringe: A Breakthrough in Pain Control
}

\author{
${ }^{1}$ Mustafa Tattan, ${ }^{2}$ Vladimir Kokovic
}

\begin{abstract}
Introduction: For over a decade of introducing the anterior middle superior alveolar (AMSA) injection, it has since majorly only been reported to show both pulpal and buccal mucosal effectiveness using a minimum of $0.9 \mathrm{ml}$ of anesthetic solution.
\end{abstract}

Aim: The aim of this study was to demonstrate the AMSA nerve block efficacy using a reduced dosage and conventional syringe, while comparing the anesthetic effectiveness of lidocaine vs mepivacaine. A primary objective was to understand the behavioral difference between the two solutions, to better adapt their application into relevant clinical scenarios, where fewer injections are administered, pain control is better understood and implemented, and collateral anesthesia altering the natural lip line is eliminated.

Materials and methods: Twelve candidates (both sexes), of ages 18 to 24, were selected. All the participants received bilateral AMSA nerve block (split-mouth technique) using the conventional syringe, where $0.6 \mathrm{ml}$ of lidocaine and mepivacaine $2 \%$ with epinephrine $1: 100,000$ were randomly administered to each side.

Results: Pulpal anesthesia on both premolar teeth showed 83.3\% success within 15 minutes with lidocaine group (LG) and mepivacaine group (MG) alike. Buccal anesthesia at both premolar teeth showed $50 \%$ success within 15 minutes with LG and $54.2 \%$ with MG. There was no difference in anesthetic effectiveness between the two groups in pulpal anesthesia ( $p \geq 0.05)$, and no significant difference in buccal mucosal anesthesia $(p \geq 0.05)$.

Conclusion: No statistically significant difference was observed between lidocaine and mepivacaine, while both expressed anesthetic effectiveness using a reduced dosage, as well as near painlessness using the conventional syringe.

Keywords: Anterior middle superior alveolar nerve block, Buccal mucosal anesthesia, Dental research, Lidocaine, Mepivacaine, Pulpal anesthesia.

\footnotetext{
${ }^{1}$ Student, ${ }^{2}$ Professor

${ }^{1}$ Department of Oral Surgery, RAK College of Dental Sciences RAK Medical and Health Sciences University, Ras al Khaimah United Arab Emirates

${ }^{2}$ Department of Oral Surgery, College of Dental Sciences, Ras al Khaimah, United Arab Emirates

Corresponding Author: Vladimir Kokovic, Professor Department of Oral Surgery, Ras al Khaimah, College of Dental Sciences, United Arab Emirates, Phone: +971551536346 e-mail: kokovicv@gmail.com
}

How to cite this article: Tattan M, Kokovic V. Anterior Middle Superior Alveolar Nerve Block Efficacy Utilizing different Anesthetic Solutions, Reduced Dosage and Conventional Syringe: A Breakthrough in Pain Control. Int J Experiment Dent Sci 2015;4(2):80-86.

\section{Source of support: Nil}

Conflict of interest: None

\section{INTRODUCTION}

Pain control has always been a crucial aspect of dentistry that has gradually shaped itself into a major foundation of dental practice. Dental science today is continuously living in an attempt to minimize any sort of pain or discomfort experienced by the patient during even the simplest dental procedures. Although pain due to the treatment can be discomforting to the patient, pain due to local anesthetic injections may also be a factor of patient anxiety and discomfort. In fact, Milogrom et al (1997) have reported that some patients will cancel, avoid, or not appear to dental appointments simply because of their aversion to local anesthetic injections and the associated numbness. ${ }^{1}$

In 1998, Friedman and Hochman, described a new anesthetic technique capable of anesthetizing both the anterior and middle superior alveolar nerves, as well as palatal nerve branches in that region, without any collateral anesthesia to the lips, face and facial muscles. ${ }^{2}$ This technique eliminates the need for multiple injections and the associated numbness that leads to patient discomfort and possible self-inflicted injury. Additionally, the alteration of the lip-line, compromising the efficiency of esthetic dental therapy, due to numbness of the soft tissues is also eliminated. ${ }^{2}$ Another recently reported advantage of this technique, is the associated postoperative pain being less than that related to the common infiltration anesthetic technique. ${ }^{3}$

The anterior middle superior alveolar (AMSA) nerve block success in anesthetizing the ipsilateral central incisors to the second premolar and the adjacent palate has been reported in several published studies since 1998. Many of the documented studies, where both pulpal and buccal mucosal anesthesia was assessed, have focused on the use of computer-controlled local anesthesia delivery 


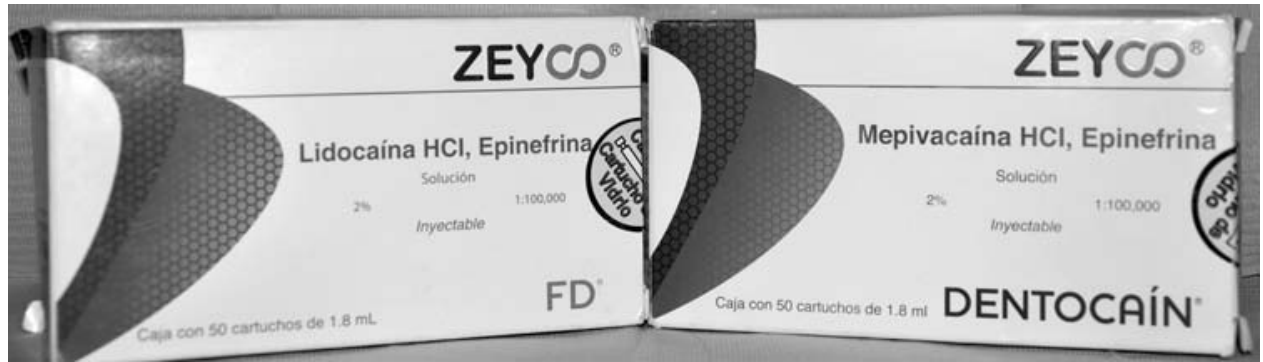

Fig. 1: Anesthetic solutions-2\% lidocaine $\mathrm{HCl}$ and $2 \%$ mepivacaine $\mathrm{HCl}$ with 1:100,000 epinephrine

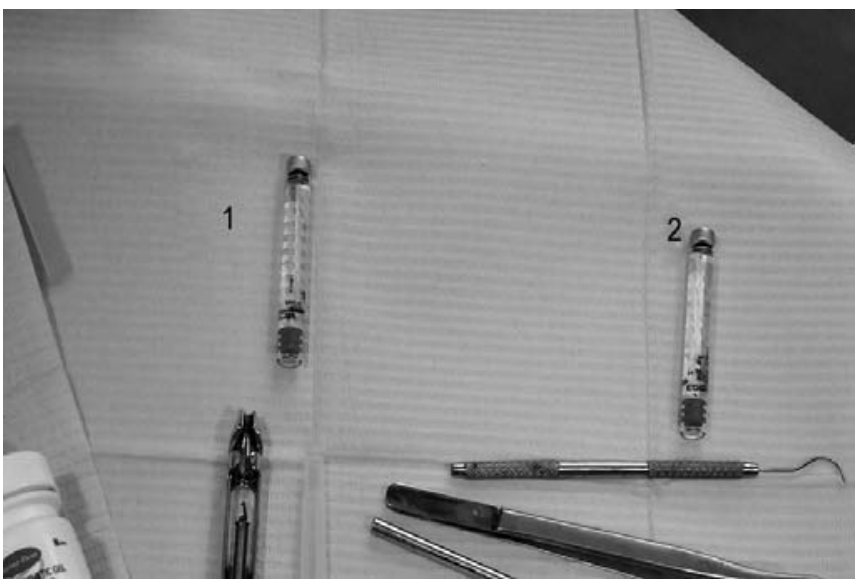

Fig. 2: Prepared anesthetic cartridges for randomization

(CCLAD) systems for pain control and/or dosages ranging from 0.9 to $1.8 \mathrm{ml} .^{4-7}$

The aim of this study was to demonstrate the efficacy of the AMSA nerve block using a reduced dosage and conventional syringe, while comparing the anesthetic effectiveness of lidocaine vs mepivacaine. A primary objective was to understand the behavioral difference between the two solutions, to better adapt their application into relevant clinical scenarios, where fewer injections can be administered, pain control is better understood and implemented, and/or collateral anesthesia affecting the natural lip line is eliminated.

\section{MATERIALS AND METHODS}

\section{Subject}

The procedure was performed in the students' clinic of RAK College of Dental Sciences, RAK Medical and Health Sciences University (RAKMHSU), Ras Al-Khaimah, UAE. To conduct this study, convenience sampling was used to collect 12 candidates (6 males and 6 females) with mean age of 20.5 years old. Ethical approval was obtained from the research ethical committee of RAKMHSU (13-2014UG-D) and all candidates were informed of the procedure with obtained written consent.

\section{Inclusion Criteria}

- Candidates with all maxillary premolars present and vital
- Candidates of American Society of Anesthesiologists class I or II

- Age group 18 to 24 .

\section{Exclusion Criteria}

- Candidates with metallic/prosthetic restorations in any of the premolars

- Candidates with acute inflammation in the palate

- Candidates with previous hypersensitivity to local anesthesia (LA)

- Smokers.

\section{Anesthetic Solutions}

Two types of local anesthetic solutions were analyzed in this study; and they were $2 \%$ mepivacaine $\mathrm{HCl}$ and $2 \%$ lidocaine $\mathrm{HCl}$, both with epinephrine 1:100,000 (ZEYCO ${ }^{\circledR}$, Mexico) (Fig. 1). Two study groups were formed correspondingly, mepivacaine group (MG) and lidocaine group (LG).

\section{Randomization}

To ensure efficient randomization and elimination of bias, the 'double-blind trial' concept was integrated into the research design. All labels on the anesthetic solution cartridges, indicating the type of solution, were removed and the cartridges were numbered randomly, to be administered into either the right or left palate accordingly (Fig. 2). Both the investigator and the supervisor were oblivious to which solution was administered into both halves of the palate until the data analysis phase of the study.

\section{Split-mouth Technique}

Additionally, for an effective comparison between lidocaine and mepivacaine to be made, both solutions need to be administered into one palate and tested. Therefore, the split-mouth technique was used to ensure this.

\section{CLINICAL PROCEDURE}

\section{Preoperative Procedure}

Every participant was first informed of the entire procedure and possible complications; and written consent 
was taken accordingly. Following this, the premolars on either sides of the maxilla were separated by cellulose strips to assess their vitality (Fig. 3). Cellulose strips were then used to separate the premolars from adjacent teeth (canine and molar) to avoid any interference in reading of tooth vitality following anesthetic administration (Fig. 4).

\section{Anesthetic Technique}

The site of injection designated for the AMSA nerve block is described as halfway between the mid-palatal suture and the crest of the gingival margin, exactly midway between the two premolars (Fig. 5). ${ }^{8}$ Prior to the AMSA injection on either side of the palate, the tissue was dried with gauze (Fig. 6) and the application of topical anesthesia followed (Fig. 7). The self-aspirating syringe (Fig. 8) was then prepared during the time period (2-3 minutes) required for the full effect of topical anesthesia to occur. Placing the patient in a supine position, the administration process was initiated and the deposition of $0.6 \mathrm{ml}$ took place along an allotted 60 seconds. As soon as the target dosage was reached, time measurement was

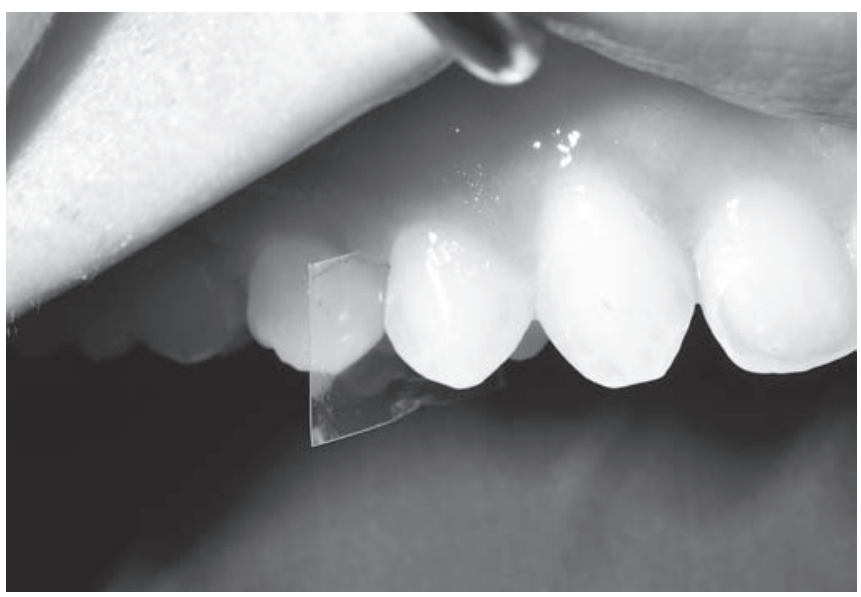

Fig. 3: Separation of premolars using a cellulose strip

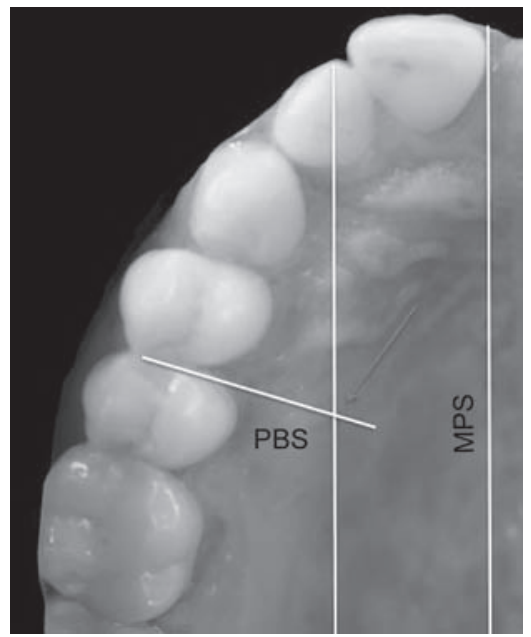

Fig. 5: Appropriate injection site for the AMSA nerve block initiated and the second injection on the opposite side of the palate took place immediately after.

\section{Investigation Parameters}

Vitality testing: Following the anesthesia procedure, the respective premolars were tested for anesthetic effectiveness on the supplying innervation. This was executed by applying Endo-Ice $\left(-50^{\circ} \mathrm{C}\right)$ on a cotton pellet and directly on the buccal surface of the tooth (Fig. 9). The patient's response was recorded at 3,5,10, and 15 minutes until negative vitality (or anesthetic effect) was achieved. Pinprick testing: Following the anesthesia procedure, the areas of buccal gingiva related to the associated premolars were tested for anesthetic effectiveness or numbness. This was performed using a diagnostic explorer (Fig. 10). The patient's response was recorded at 3, 5, 10 and 15 minutes until negative vitality (or anesthetic effect) was achieved. Pain assessment: Following the anesthesia and data collection procedures, the level of pain experienced by the candidate was recorded using visual analog scale (VAS) and pain rating score (PRS). Visual analog scale was a measure of

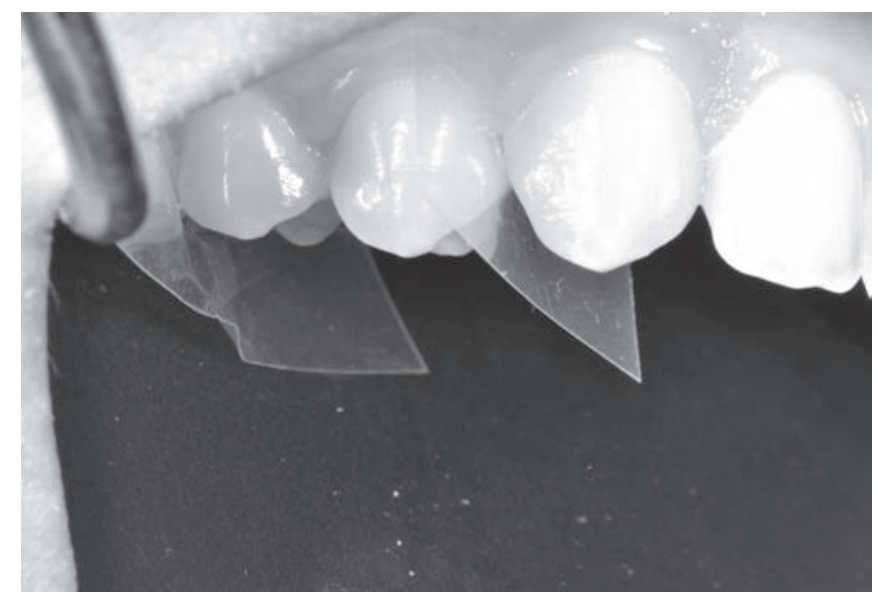

Fig. 4: Separation of premolars from adjacent teeth (canine and molar)

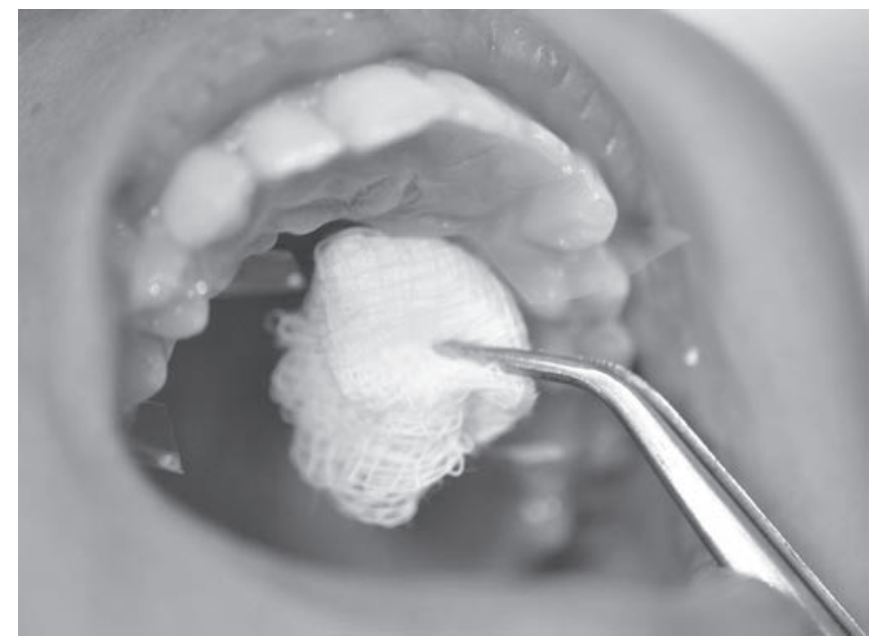

Fig. 6: Drying of the palatal site of injection using gauze 


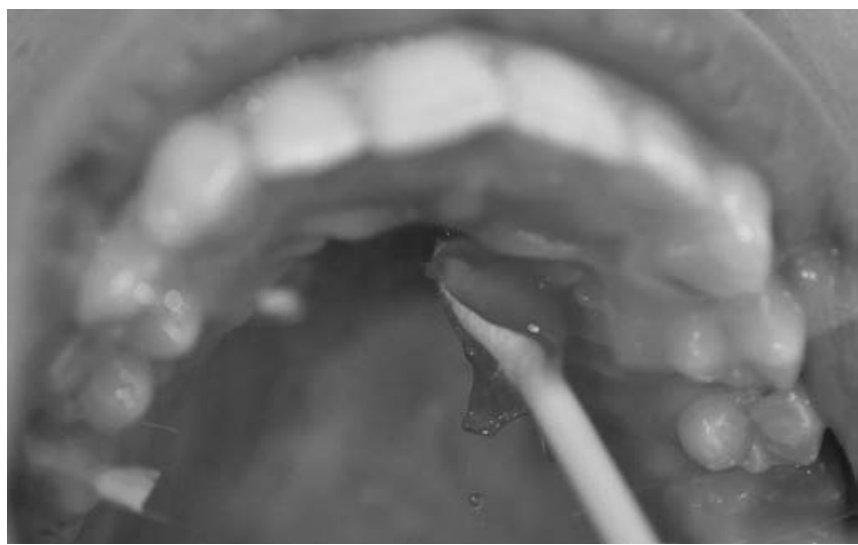

Fig. 7: Application of topical anesthesia at the site of injection

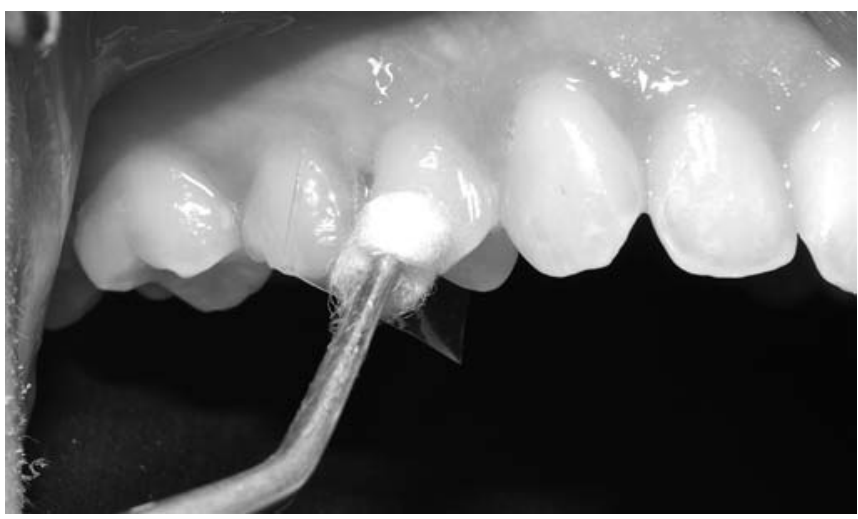

Fig. 9: Vitality testing

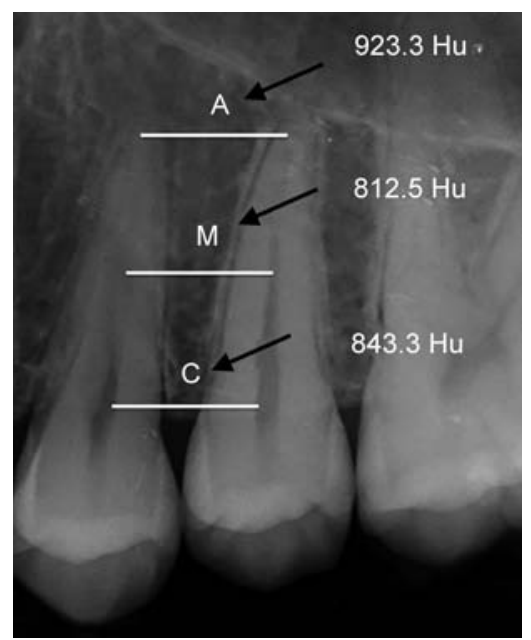

Fig. 11: Radiograph with bone density analysis (DfW 2.7 software)

pain intensity based on a marking drawn by the participant on a $10 \mathrm{~cm}$ line. Pain rating score included 'severe pain', 'moderate pain', 'slight pain' and 'no pain' for each participant to choose from depending on their experience. Bone density analysis: Following the anesthesia and data collection procedures, two periapical radiographs were taken for each side of the palate from each candidate. The density of the bone between each two premolars was identified using the X-ray software, DfW 2.7. The average of the apical, middle, and cervical portions was calculated for each participant (Fig. 11).

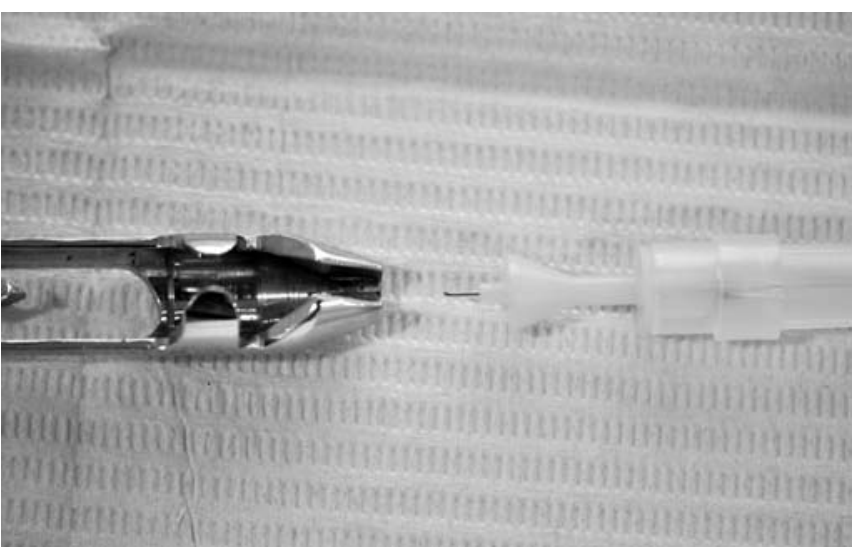

Fig. 8: Self-aspirating syringe

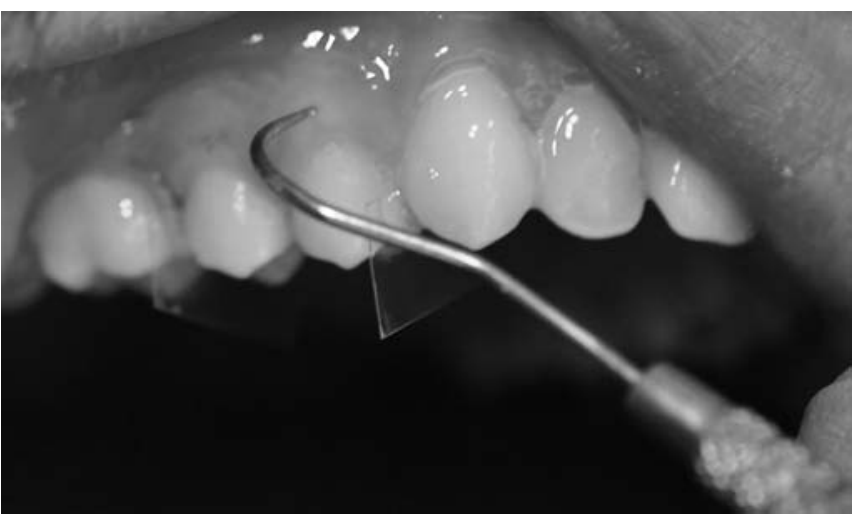

Fig. 10: Pinprick testing

\section{STATISTICAL ANALYSIS}

All data were analyzed by descriptive methods Statistical Package for the Social Sciences (SPSS 18.0, Chicago, IL, USA). Analyses of variance (ANOVA) were applied to detect differences in the effectiveness of anesthetic success between lidocaine and mepivacaine in this maxillary anesthetic technique. The sample size was statistically determined. The level of significance chosen in all statistical tests was at $\mathrm{p}<0.05$.

\section{RESULTS}

\section{Vitality Testing}

Both MG, as well as LG showed 83\% (20) anesthetic success within 15 minutes on tooth vitality, showing no statistical significance when t-test was used $(\mathrm{p}<0.05)$. Additionally, LG showed 58 (14), 50 (12) and 17\% (4) anesthetic success within 10, 5 and 3 minutes respectively. Mepivacaine group showed 83 (20), 58 (14) and 38\% (9) anesthetic success within 10, 5 and 3 minutes respectively (Graph 1).

\section{Pinprick Testing}

Within 15 minutes, MG showed 54\% (13) anesthetic effectiveness on buccal mucosa in the premolar region, while LG showed 50\% (12) anesthetic effectiveness in the same. Also, LG showed 25\% (6) effectiveness 


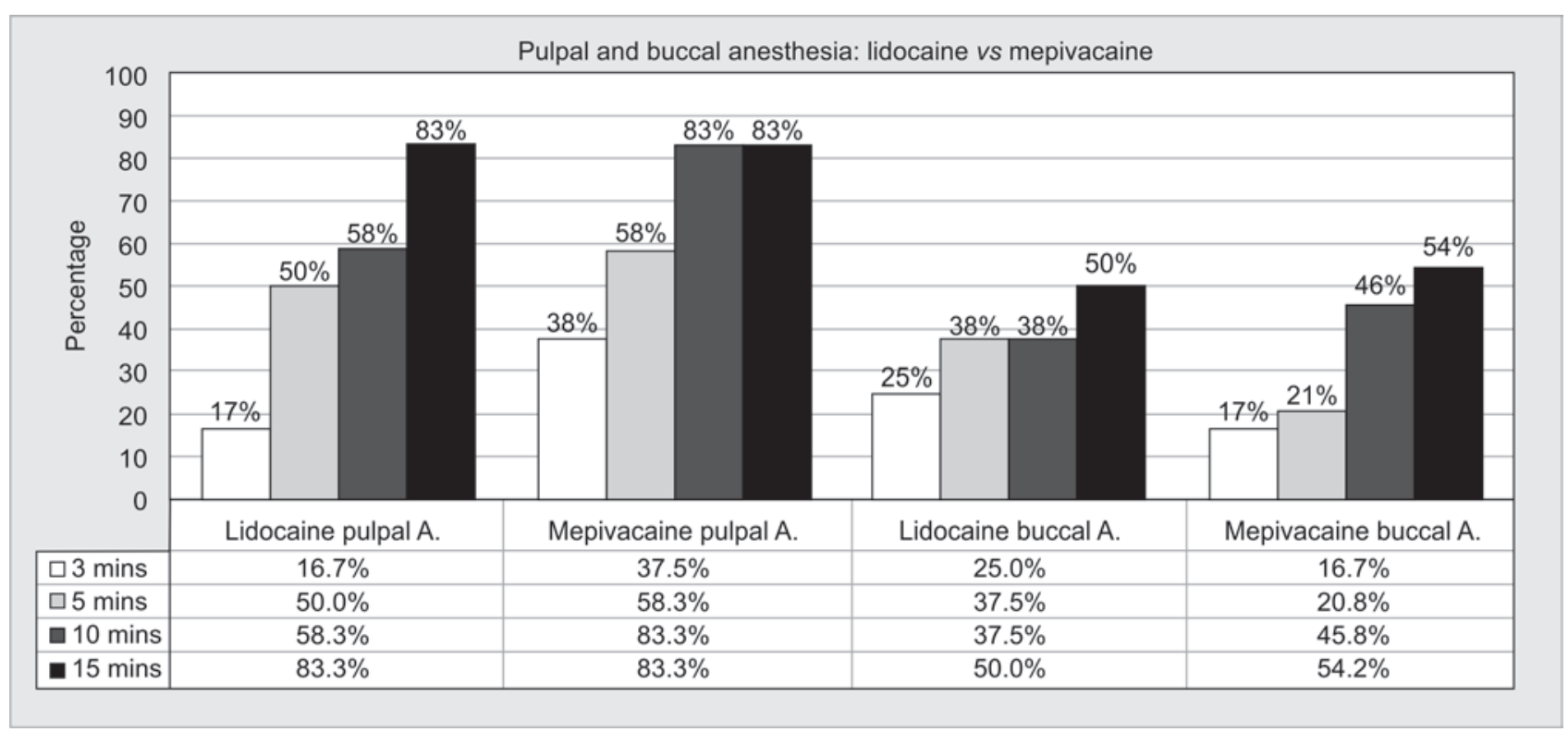

Graph 1: Result of the anesthesia effectiveness on pulu and buccal mucosa during the observation period

within 3 minutes and 38\% (9) within 5 and 10 minutes. Mepivacaine group, however, showed 46 (11), 21 (5) and $17 \%$ (4) anesthetic effectiveness within 10,5 and 3 minutes respectively (Graph 1).

\section{Pain Assessment}

The VAS showed an average score of $1.31+0.48 \mathrm{~cm}$ for all participants who received the AMSA injection. And the PRS showed that 58 and $42 \%$ of all participants reported 'slight pain' and 'no pain', respectively, while no patients reported 'severe pain' or 'moderate pain'.

\section{Bone Density Analysis}

The average bone density in all participants was 859.7 $\pm 287.6 \mathrm{HU}$ (400 minutes, 1210 max) or class II bone density based on the Lekholm and zarb bone density classification. ${ }^{9}$ No statistically significant correlation was observed between bone density and anesthetic effectiveness in this study.

\section{DISCUSSION}

Ever since the very first recommendation of the AMSA injection (1998), ${ }^{2}$ many published studies have tested this technique, focusing on its effectiveness and the pain control simultaneously required along with it. The focus on pain control was often associated with the use of CCLAD. ${ }^{2,5,10,11}$ However, costliness is an associated disadvantage which retards the ability for many clinicians to practically implement this technique in their daily practice. This study reinforces the practicality of the conventional syringe through the results obtained from the undertaken pain assessments, where neither 'severe' nor 'moderate' pain was reported from the entire study sample during the injection. These findings of near painlessness associated with the use of the conventional syringe have been observed before. ${ }^{12}$

Following the demonstration of the independence of a painful AMSA injection with the use of a conventional syringe, other possibly causative factors associated with pain in palatal injections are magnified. A probable cause, that has been previously mentioned, is the pressure buildup in the limited space of the palatal mucoperiosteum. ${ }^{11}$ This is commonly associated with pressure-inducing dosages of solution administered in this area. Majority of the studies previously published around the AMSA technique have procedures performed with the use of dosages ranging from 0.9 to $1.8 \mathrm{ml}$ when testing for both pulpal and buccal mucosal anesthetic effectiveness. ${ }^{2,3,3,6,7,12}$ The results of this study display the reduction of administered solution $(0.6 \mathrm{ml})$, while still maintaining the anesthetic effectiveness of both tooth vitality $(83 \%)$, as well as buccal mucosa (50-54\%) within 15 minutes.

Another important factor of pain control, which has been mentioned as a disadvantage of the AMSA injection, ${ }^{11}$ is the time of administration. The duration required for the deposition of a full cartridge of anesthesia to induce a minimal amount of pain is 4 minutes. ${ }^{11}$ However, with a reduced dosage necessary for sufficient anesthetic effectiveness, such as $0.6 \mathrm{ml}$ of solution, the time of administration is, in turn, reduced to only 60 seconds, as performed in this study. Both patient and operator comfort were demonstrated with these reductions in dosage and technique duration.

Among the many advantages regarding the AMSA injection, no vasoconstrictor affecting the buccal gingiva 

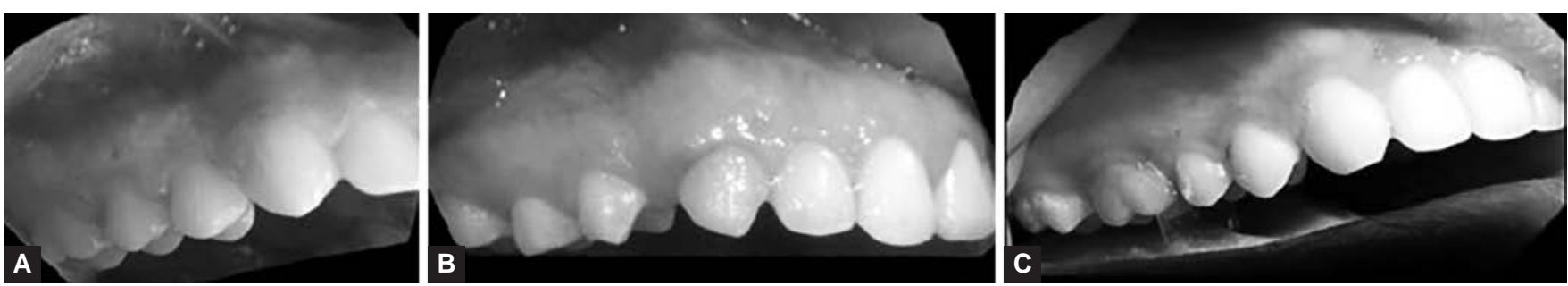

Figs 12A to C: Results of the anesthetic effectiveness on the pulp and buccal mucosa during the observation period

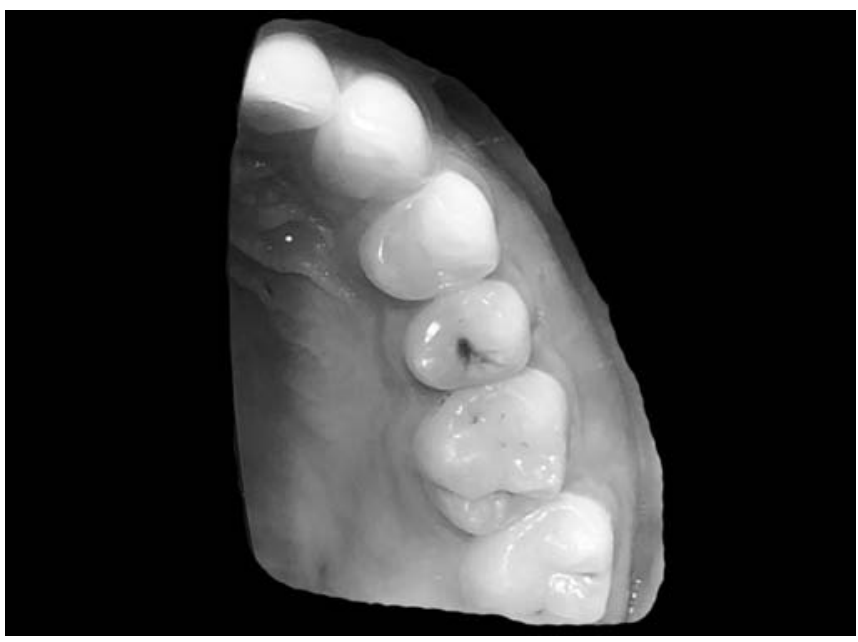

Fig. 13: Case of exostosis associated with negative anesthesia

and outstanding blood supply maintained for nourishment of a buccal connective tissue graft were mentioned. ${ }^{11}$ However, while undergoing this study, areas of ischemia on the buccal gingiva were observed in $25 \%$ of the cases following the injection technique (Figs 12A to C). The ischemia clearly seen on the buccal gingiva is indicative of vasoconstriction occurring as a result of the anesthetic solution. Therefore, these findings invalidate the mentioned AMSA nerve block advantage regarding no vasoconstrictor affecting the buccal gingiva, jeopardizing the success of the connective tissue graft.

Bone density was not found to have any statistically significant correlation with anesthetic effectiveness. However, a case of exostosis (Fig. 13) was present within the study sample where negative buccal mucosal anesthesia in both study groups and negative pulpal anesthesia in LG were observed.

\section{CONCLUSION}

This study has advanced the practical understanding and implementation of the AMSA injection technique into numerous clinical scenarios with more efficient anesthetic effectiveness, as well as improved pain control. Efficacious pulpal and buccal mucosal anesthesia with a reduced dosage $(0.6 \mathrm{ml})$ of different anesthetic solutions (lidocaine and mepivacaine) using the conventional syringe was demonstrated, along with outstanding patient and operator comfort. There was no statistical significance between lidocaine and mepivacaine in anesthetic effectiveness. And, bone density was not observed to have a statistically significant correlation with anesthetic effectiveness. Despite the quite high percentage of anesthetic success demonstrated in this study on the premolars within 15 minutes, further investigations can be performed, where the observing time is extended and the anterior teeth are included in the observation. Dentistry is varying with induction of modern science to practice dentistry. ${ }^{13}$

\section{ACKNOWLEDGMENTS}

The authors would like to thank ZEYCO ${ }^{\circledR}$ for supporting the study with materials. Also, the principal author would like to directly express his gratitude to close colleagues for their support, with special thanks to Aya Bakkour. The college's support was also very much appreciated.

\section{REFERENCES}

1. Milogram P, Coldwell SE, Getz T, Weinstein P, Ramsay DS. Four dimensions of fear of dental injections. J Am Dent Assoc 1997;128(6):756-762.

2. Friedman M, Hochman M. The AMSA injection: a new concept for local anesthesia of maxillary teeth using a computer-controlled injection system. Quintessence Int 1998; 29(5):297-303.

3. Shirmohammadi A, Faramarzi M, Lafzi A, Kashefimehr A, Malek S. Comparison of pain intensity of anterior middle superior alveolar injection with infiltration anesthetic technique in maxillary periodontal surgery. J Periodon Imp Sci 2012;42(2):45-49.

4. Friedman M, Hochman M. P-ASA block injection: a new palatal technique to anesthetize maxillary anterior teeth. J Anesthetic Dentistry 1999;11(2):63-71.

5. Fukayama H, Yoshikawa F, Kohase H, Umino M, Suzuki N. Efficacy of anterior and middle superior alveolar anesthesia using a new injection system: the wand. Quintessence Int 2003;34(7):537-541

6. Holtzclaw D, Toscano N. Alternative anesthetic technique for maxillary periodontal surgery. J Periodon 2008;79(9): 1769-1772.

7. Lee S, Reader A, Nusstein J, Beck M, Weaver J. Anesthetic efficacy of the anterior middle superior alveolar injection. Anesth Prog 2004;51(3):80-89.

8. Malamed SF. Handbook of Local Anesthesia. Missouri, US: Elsevier. 6th ed. 2013; p. 188-218. 
9. Lekholm U, Zarb GA. Patient selection and preparation. In: Brånemark PI, Zarb GA, Albrektsson T, editors. TissueIntegrated Prostheses: Osseointegration in Clinical Dentistry. Chicago: Quintessence; 1985. p. 199-209.

10. Saloum FS, Baumgartner JC, Marshall G, Tinkle J. A clinical comparison of pain perception to the wand and a traditional syringe. Oral Surg Oral Med Oral Pathol Oral Radiol Endod 2000;89(6):691-695.
11. Alam M, Mohan V, Balaji A. Anterior middle superior alveolar injection: a boon to maxillary periodontal surgery. J Clin Diag Res 2011;5(3):675-678.

12. Velasco I, Soto R. Anterior and middle superior alveolar nerve block for anesthesia of maxillary teeth using conventional syringe. Dent Res J (Isfahan) 2012;9(5):535-540.

13. Saini R. Ozone therapy in dentistry: a strategic review. J Nat Sc Biol Med 2011;2(2):151-153. 